\title{
Spectroscopic Measurements in Patients with Completely Ruptured Anterior Cruciate Ligament before and after RegentK and Physiotherapy
}

\author{
Daniela Litscher ${ }^{a-c}$ Gerhard Litscher ${ }^{a-c}$ Michael Ofner ${ }^{\mathrm{e}}$ \\ Ingrid Gaischek $^{\mathrm{a}-\mathrm{c}}$ Daniela-Eugenia Malliga ${ }^{\mathrm{d}}$
}

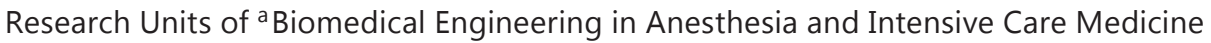
and ${ }^{\mathrm{b}}$ Complementary and Integrative Laser Medicine, ${ }^{\mathrm{c}} \mathrm{TCM}$ Research Center Graz, and dDivision of Cardiac Surgery, Department of Surgery, Medical University of Graz, Graz, and

e Department of Sports Physiology, University of Vienna, Vienna, Austria

\section{Key Words}

RegentK · Khalifa therapy · Physiotherapy · Anterior cruciate ligament · Near-infrared spectroscopy $\cdot$ Regional oxygen saturation

\section{Abstract}

Background: The goal of this study was to investigate acute effects of a special kind of manual therapy developed by Mohamed Khalifa (RegentK) compared to standard physiotherapy on regional oxygen saturation $\left(\mathrm{rSO}_{2}\right)$ of knee tissues in patients with completely ruptured anterior cruciate ligament $(\mathrm{ACL})$. Methods: Twenty patients were enrolled in this study and randomly assigned to receive either RegentK (group $A ; n=10,8$ female, 2 male, mean age $\pm S D$ $31.3 \pm 8.5$ years) or physiotherapy (group $\mathrm{B} ; \mathrm{n}=10,6$ female, 4 male, mean age $34.8 \pm 10.2$ years). The values of $\mathrm{rSO}_{2}$, assessed using near-infrared spectroscopy, were registered anterolaterally and anteromedially on both the injured and the healthy (control) knee. Results: $\mathrm{rSO}_{2}$ increased significantly ( $p$ < 0.001) after RegentK on both registration sites of the injured knee, whereas after physiotherapy only the anterolateral side showed significant increases. Interestingly, we found significant increases in $\mathrm{rSO}_{2}$ also on the control knee after RegentK; in group $B$, these results were insignificant. Conclusion: We conclude that manual therapy influences the hemodynamics of muscles and deeper structures. The results will also serve to quantify the effects of nonpharmacological and nonsurgical interventions (e.g. RegentK) on the microvascular circulation in deep tissue after a complete rupture of the $\mathrm{ACL}$. 


\section{Introduction}

Every year, more than 10,000 Austrians suffer an injury of the cruciate ligament, most of them during sports, especially skiing or playing football. According to Statistics Austria 2012, more than 8,600 of these ligaments were reconstructed surgically. Recent studies, however, have shown that a ruptured cruciate ligament can regrow even without surgical intervention. Normally this would take many months, but sometimes the healing process can be accelerated. In the small village Hallein (close to Salzburg) in Austria, Mohamed Khalifa has been treating knee ligament injuries for more than 30 years with a noninvasive pressure therapy, the so-called regeneration therapy after Khalifa (RegentK). His patients are not only top athletes but also surgeons [1-3]. However, at the moment, Mohamed Khalifa has no students, and therefore the therapy cannot be performed by other therapists. Our research tries to investigate the underlying mechanisms in order to create a new integrative treatment approach.

There are many different measurement methods in physical medicine and rehabilitation, and instrumental assessment is becoming increasingly important in clinical practice for the quantification of the effects of different rehabilitation methods. Near-infrared spectroscopy (NIRS) is one of these methods. It was described for the first time by Jöbsis in Science in 1977 [4]. It is a noninvasive technology, and the multichannel monitoring equipment led to numerous clinical applications. In rehabilitation medicine, NIRS is also used for investigating changes in oxygen saturation of the lower extremities; however, there are only few reliable basic and clinical data available $[2,5]$.

The goal of this randomized controlled study was to investigate acute effects of RegentK compared to standard physiotherapy on regional oxygen saturation $\left.\mathrm{rSO}_{2}\right)$ of knee tissues in patients with completely ruptured anterior cruciate ligament (ACL) using new modern technical equipment.

\section{Patients and Methods}

\section{RegentK}

Khalifa therapy has been reported in detail in a previous paper [2]:

'Khalifa therapy is described as functional-pathological [6]. In this approach, function is the primary concern, not anatomy. The most important thing is not the ruptured ligament itself, but its function/ dysfunction. Khalifa therapy restores the function of the knee in a natural way. During the 60-90 min of his manual therapy, he applies pressure to the injured knee in order to activate the self-healing processes of the human body, using his hands as an instrument for both measurement and therapy. Over periods of varying length, he applies increasing pressure on a spot before moving on to the next spot. The frequency of pressure application depends on the patient's physiological reaction. The force of the pressure is not comparable to that normally used in acupressure in Traditional Chinese Medicine [5]; it is much higher [...]. Mohamed Khalifa's method is based on manual pressure of varying frequency and does not damage the body, but supports it in its own natural healing activities. If one cuts through an elastic band and sews it together again, one cannot expect it to be as elastic at the stitching point as it was before. It is the same with human ligaments, and if the elasticity is disrupted anywhere in the human body, the whole system is affected $[6,7] . '$

Physiotherapy

Physiotherapeutic intervention in order to mobilize/activate the knee joint included basic techniques of different myofascial treatment methods. Focus was put on the myofascial structures of tissues surrounding the knee joint; the upper part of the body and the upper extremities were not included in the treatment. Two basic techniques were used during the control intervention: (1) a static manual treatment, i.e. pressing trigger points usually to be found in the muscle belly (typically in the m. quadriceps, $\mathrm{m}$. biceps femoris, $\mathrm{m}$. popliteus, or m. gastrocnemius), and (2) static-dynamic stretching with intermuscular techniques in the areas of the tractus iliotibialis, the medial femoral muscles directed toward the knee or between (and in) the muscle bellies of the $\mathrm{m}$. gastrocnemius. 
Table 1. Demographic data of both patient groups

\begin{tabular}{|c|c|c|}
\hline \multicolumn{2}{|c|}{ DOI: 10.1159/000365105 } & $\begin{array}{l}\text { (c) } 2014 \text { S. Karger AG, Basel } \\
\text { www.karger.com/imi }\end{array}$ \\
\hline \multicolumn{3}{|c|}{$\begin{array}{l}\text { Litscher et al.: Spectroscopic Measurements in Patients with Completely Ruptured } \\
\text { Anterior Cruciate Ligament before and after RegentK and Physiotherapy }\end{array}$} \\
\hline & Group A (RegentK) & Group B (physiotherapy) \\
\hline Age, years & $31.3 \pm 8.5$ & $34.8 \pm 10.2$ \\
\hline Range & $20-43$ & $19-47$ \\
\hline Height, $\mathrm{cm}$ & $170.5 \pm 10.4$ & $174.8 \pm 9.7$ \\
\hline Weight, kg & $67.7 \pm 12.2$ & $66.1 \pm 12.0$ \\
\hline $\operatorname{Sex}(f / m)$ & $8 / 2$ & $6 / 4$ \\
\hline \multicolumn{3}{|l|}{ Cause of injury } \\
\hline Skiing & 8 & 9 \\
\hline Household & 1 & - \\
\hline Volleyball & 1 & 1 \\
\hline \multicolumn{3}{|l|}{ Injured side } \\
\hline Left & 5 & 4 \\
\hline Right & 5 & 6 \\
\hline
\end{tabular}

Data are given as means \pm SD or number of patients.

Both techniques were performed at the patient's individual pain threshold, and static trigger points were pressed 3-5 times (for a maximum of $30 \mathrm{~s}$ each) until the tension and pain subsided. The myofascial longitudinal and crosswise stretchings were also repeated 3-5 times, slowly and dynamically.

Before and during the treatment (duration about $60 \mathrm{~min}$ ), the patients were informed about potential physiologic reactions due to the interventions [8-12].

\section{Patients}

Twenty patients were randomly assigned to the two intervention groups (group A: RegentK; group B: physiotherapy). The demographic data of the two groups can be seen in table 1.

Inclusion criteria were as follows: unilateral complete rupture of the ACL (less than 14 days ago), verified by magnetic resonance imaging; age 18-49 years; normal body weight: BMI 18-25; regular exercise level; knee instability: experienced at least one giving-way, and dysfunction: reduced or inhibited knee range of motion. Further, the following exclusion criteria were used: preceding surgical intervention (including arthroscopy) on the injured knee; metabolic disorders such as diabetes mellitus, and autoimmune diseases.

The patients were informed about the nature of the investigation as far as the study design allowed it and were not paid for their participation. The study was approved by the Ethics Committee of the University of Salzburg, Austria (21-232 11-12 sbg + amendment) and registered at clinicaltrials.gov (ID No. NCT01762371). All participants provided written informed consent.

\section{Evaluation Parameters}

The NIRS measurements were performed using a four-channel INVOS 5100C Oximeter (Somanetics, Troy, Mich., USA). The principle of this system is based on the NIRS technology, a noninvasive method for measuring regional oxygenation through the intact skin [13]. Near-infrared light (730 and $805 \mathrm{~nm}$ ) is emitted through the skin, and, after passing different kinds of tissue (muscle and bone), the returned light is detected at two distances from the light source $(3$ and $4 \mathrm{~cm}$ ). Based upon this principle, the spectral absorption of blood in deeper structures $(2-4 \mathrm{~cm})$ can be determined and defined as $\mathrm{rSO}_{2}[5,13]$. The different tissues of the human body have different absorption characteristics, and, therefore, it is not easy to define in which kind of tissue the $\mathrm{rSO}_{2}$ value is measured [2].

\section{Procedure}

The NIRS measurement procedure was the same for both patient groups. Approximately $3 \mathrm{~h}$ before starting the measurement, both legs were shaved. The skin was then cleaned with the enclosed skin prep pad and dried with a gauze pad. After removing the protective backing label from the adhesive side of the sensor, four sensors were applied below the right and the left lateral side of the patella of both legs. Calibration was performed automatically. To minimize external light influence, the knees were covered with a dark surgical 
Litscher et al.: Spectroscopic Measurements in Patients with Completely Ruptured

Anterior Cruciate Ligament before and after RegentK and Physiotherapy

Fig. 1. Box plot of changes in $\mathrm{rSO}_{2}$ on the injured knee after RegentK (ends of boxes: 25th and 75th percentiles; line at the median; error bars: 10th and 90th percentiles).

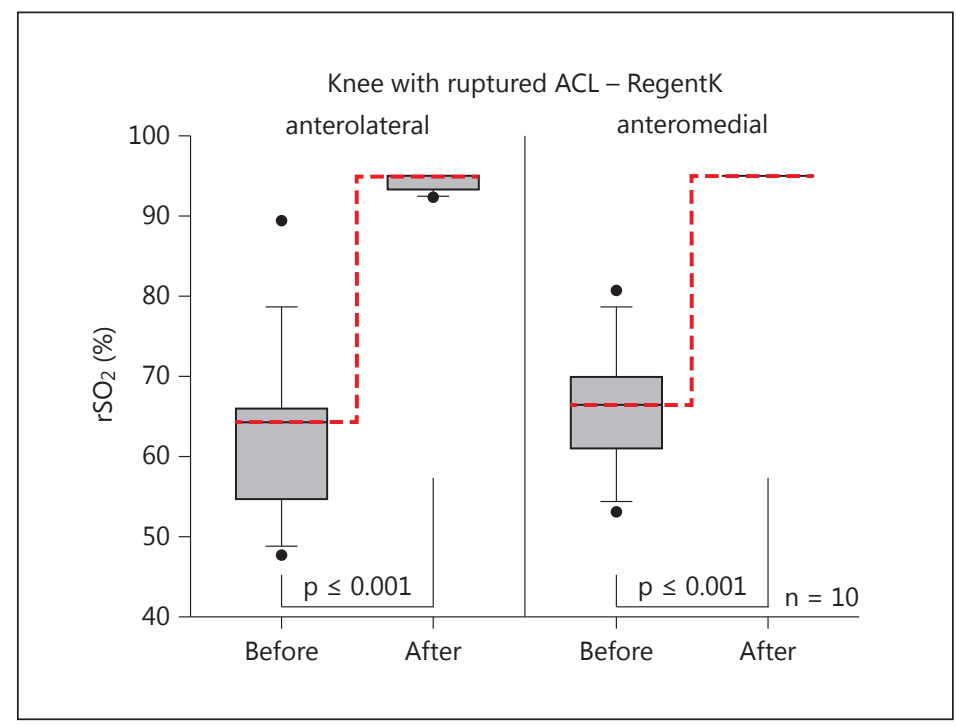

cloth during the recording procedure. The $\mathrm{rSO}_{2}$ data were recorded before the start and after the end of the respective manual therapy (three readings each within approximately $2 \mathrm{~min}$; for graphical presentation, only the mean values 'before' and 'after' were chosen).

All patients were investigated in a supine position under similar conditions. The parameter $\mathrm{rSO}_{2}$ was simultaneously measured at four sites: laterally and medially at both knees, the injured one and the healthy one (control knee; this knee did not receive any therapy or manual manipulation; only the measurement sensors were applied). To avoid a potential technical recording bias, the four channels of the equipment were used in a randomized order as done in a previous study [2].

Statistical Analysis

The $\mathrm{rSO}_{2}$ values of both legs were tested with one-way repeated-measures ANOVA (SigmaPlot 12.0; Systat Software Inc., Chicago, Ill., USA). The Tukey test was used for post hoc analysis. The level of significance was defined as $\mathrm{p}<0.05$.

\section{Results}

In all patients who fulfilled the inclusion criteria, neither the initial conditions (knee instability and dysfunction) nor the demographic data showed significant differences between group A and group B. All 20 patients completed the study procedure, and the technological NIRS measurements could be performed without problems.

Figure 1 shows the highly significant $(\mathrm{p}<0.001)$ increase of $\mathrm{rSO}_{2}$ values after RegentK on the injured knee. It has to be mentioned that this increase was a little bit more pronounced on the anteromedial side of the injured knee (fig. 1, right).

The $\mathrm{rSO}_{2}$ values on the injured knee before and after physiotherapy are shown in figure 2. The baseline values before treatment were within the same range as those in group A. However, the increase after physiotherapy was far less than after RegentK. The level of statistical significance was reached on the anterolateral side $(\mathrm{p}=0.015)$ but not on the anteromedial side of the injured knee.

Figures 3 and 4 show the values of the NIRS oximeter on the control (healthy) knee. It is interesting that the baseline values before therapy were lower than those of the injured knee. In addition, there was a highly significant increase on both measurement sites (anterolateral 
Integrative

Medicine

International

Fig. 2. Changes in $\mathrm{rSO}_{2}$ on the injured knee before and after physiotherapy. For further explanations, see legend to figure 1 .

Fig. 3. Changes in $\mathrm{rSO}_{2}$ on the healthy control knee before and after RegentK. For further explanations, see legend to figure 1 .

Fig. 4. Changes in $\mathrm{rSO}_{2}$ on the healthy control knee before and after physiotherapy. For further explanations, see legend to figure 1.
Litscher et al.: Spectroscopic Measurements in Patients with Completely Ruptured Anterior Cruciate Ligament before and after RegentK and Physiotherapy
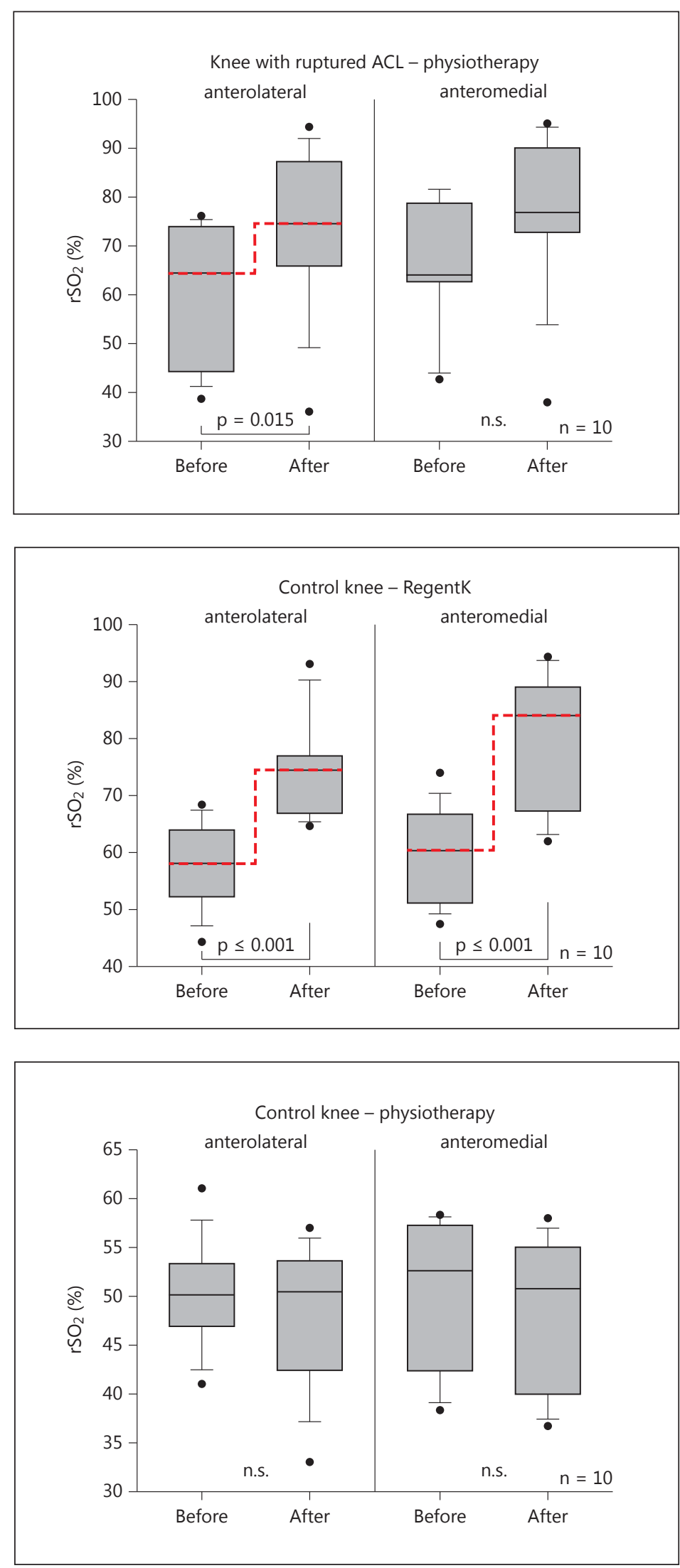
and anteromedial) of the healthy knee after RegentK. Figure 4 summarizes the $\mathrm{rSO}_{2}$ results of the control knee before and after physiotherapy. No significant changes were found anterolaterally and anteromedially.

Within this randomized controlled trial, the function of all 10 knees with a ruptured ACL was restored after 60-90 min of RegentK, but not after about 60 min of physiotherapy. All patients in group A were able to bend their knees immediately after the end of RegentK. The biomechanical data will be described in future publications by another research team. In group B, some success could also be seen, but not to the same extent as in group A.

\section{Discussion}

The first study to report an immediate close-to-normal functional restoration after one nonsurgical intervention was published recently [1]. Three months after the intervention, $47 \%$ of the patients with an initially MRI-verified complete ACL rupture showed an end-toend continuous ACL, again MRI-verified [1].

An increase in tissue blood flow is one of the most acknowledged potential effects of massage [14]. Up to now, research studies investigating this phenomenon are rare, inconsistent and inconclusive [15]. Many studies in the field of manual therapy and regeneration employed laser Doppler techniques $[16,17]$. The problem is that laser Doppler can only estimate blood flow at the skin level. On the other hand, there is also the possibility of measuring with Doppler ultrasound, but this method is not sensitive to blood flow in the smaller vasculature $[14,18]$. However, there are also two preliminary studies from our research team at the Medical University of Graz investigating these effects using noninvasive methods. One of these studies deals with NIRS measurements before and after acupressure at an acupoint on the knee [5], and the other one is a preliminary study from the first phase of the current research project on RegentK [2].

In our opinion - and also in that of others - NIRS may be a more accessible measurement option for manual therapy and researchers focusing on regeneration. The method provides dynamic information about oxy- and deoxyhemoglobin concentrations and blood oxygen saturation in deeper tissues [19].

The instrument used in our study is a commercial one, but many prototypes with different functions are also available. One system will measure massage and manual therapy effects on skeletal muscle hemodynamics using a novel hybrid near-infrared optical instrument that combines a commercial NIRS system with a custom-made one for the simultaneous measurement of muscle blood flow and oxygenation [14]. The principle of this hybrid instrument is described elsewhere [20].

The Swiss Medical Board recommends that ACL ruptures should preferably be treated with physiotherapy as no significant differences in outcomes were found between a surgical and a conservative treatment group [21]. However, the difference in costs is huge. It is estimated that more than CHF 35 million could be saved per year by applying conservative treatment for ruptured ACLs [21]. Using conventional physiotherapy usually requires several treatment sessions. RegentK, on the other hand, consists of only one treatment session.

In conclusion, spontaneous healing of ACL ruptures is possible within 3 months of RegentK [1], and basic research on its mechanisms is now proceeding at full speed. Based on the results of the current study in patients after RegentK or physiotherapy, we conclude that manual therapy influences the hemodynamics of muscles and deeper structures. These results are in accordance with the only previous measurements on this topic [2,5]. They will 
Litscher et al.: Spectroscopic Measurements in Patients with Completely Ruptured

Anterior Cruciate Ligament before and after RegentK and Physiotherapy

serve as a tool to quantify the effects of nonpharmacological and nonsurgical interventions (e.g. RegentK) on microvascular circulation in deep tissue after a complete rupture of the ACL.

\section{Acknowledgments}

This study forms part of the project 'Interdisciplinary evaluation of acute effects of the Khalifa therapy in patients with ruptured anterior cruciate ligament in the knee' (phase 2; data acquisition in January 2014) (project part: Biomedical engineering and analyses focused on NIRS - thermography and Doppler flowmetry). The study was supported by the Forschungsförderungsverein der Erkenntnisse von Mohamed Khalifa. The authors are grateful to Mohamed Khalifa for his cooperation and for treating the 10 patients. The authors would also like to thank Frank Schreier, MSc (General Manager, Schreier Business Consulting, Salzburg, Austria).

\section{Disclosure Statement}

The authors declare that there is no conflict of interest concerning the publication of this article. The authors assume responsibility for the overall content and integrity of this paper.

\section{References}

-1 Ofner M, Kastner A, Wallenböck E, Pehn R, Schneider F, Gröll R, Szolar D, Walach H, Litscher G, SandnerKiesling A: Manual Khalifa therapy improves functional and morphological outcome of patients with anterior cruciate ligament rupture in the knee: a randomized controlled trial. Evid Based Complement Alternat Med 2014;2014:462840.

-2 Litscher G, Ofner M, Litscher D: Manual Khalifa therapy in patients with completely ruptured anterior cruciate ligament in the knee: first results from near-infrared spectroscopy. N Am J Med Sci 2013;5:320-324.

-3 Litscher G, Ofner M, Litscher D: Manual Khalifa therapy in patients with completely ruptured anterior cruciate ligament in the knee: first preliminary results from thermal imaging. N Am J Med Sci 2013;5:473-479.

$>4$ Jöbsis FF: Noninvasive, infrared monitoring of cerebral and myocardial oxygen sufficiency and circulatory parameters. Science 1977;198:1264-1267.

-5 Litscher G, Ofner M, He W, Wang L, Gaischek I: Acupressure at the meridian acupoint Xiyangguan (GB33) influences near-infrared spectroscopic parameters (regional oxygen saturation) in deeper tissue of the knee in healthy volunteers. Evid Based Complement Alternat Med 2013;2013:370341.

6 Niederführ G: Torn Ligaments? A Slipped Disc? Operations No Longer Necessary! Norderstedt, Books on Demand, 2001.

7 Niederführ G: Healing instead of repairing. Bad Honnef, K.H. Bock, 2010.

-8 Trampas A, Kitsios A, Sykaras E, Symeonidis S, Lazarou L: Clinical massage and modified Proprioceptive Neuromuscular Facilitation stretching in males with latent myofascial trigger points. Phys Ther Sport 2010; 11:91-98.

-9 Grieve R, Cranston A, Henderson A, John R, Malone G, Mayall C: The immediate effect of triceps surae myofascial trigger point therapy on restricted active ankle joint dorsiflexion in recreational runners: a crossover randomised controlled trial. J Bodyw Mov Ther 2013;17:453-461.

10 Henry R, Cahill CM, Wood G, Hroch J, Wilson R, Cupido T, Vandenkerkhof E: Myofascial pain in patients waitlisted for total knee arthroplasty. Pain Res Manag 2012;17:321-327.

11 Bronfort G, Haas M, Evans R, Leininger B, Triano J: Effectiveness of manual therapies: the UK evidence report. Chiropr Osteopat 2010;18:3.

$\$ 12$ Zalta J: Massage therapy protocol for post-anterior cruciate ligament reconstruction patellofemoral pain syndrome: a case report. Int J Ther Massage Bodywork 2008;1:11-21.

13 Litscher G, Schwarz G (eds): Transcranial Cerebral Oximetry. Lengerich, Pabst Science Publishers, 1997.

14 Munk N, Symons B, Shang Y, Cheng R, Yu GQ: Noninvasively measuring the hemodynamic effects of massage on skeletal muscle: a novel hybrid near-infrared diffuse optical instrument. J Bodyw Mov Ther 2012;16:22-28.

15 Weerapong P, Hume PA, Kolt GS: The mechanisms of massage and effects on performance, muscle recovery and injury prevention. Sports Med 2005;35:236-256.

$\$ 16$ Tochikubo O, Ri S, Kura N: Effects of pulse-synchronized massage with air cuffs on peripheral blood flow and autonomic nervous system. Circ J 2006;70:1159-1163. 
17 Wiltshire EV, Poitras V, Pak M, Hong T, Rayner J, Tschakovsky ME: Massage impairs postexercise muscle blood flow and 'lactic acid' removal. Med Sci Sports Exerc 2010;42:1062-1071.

-18 Yu G, Durduran T, Lech G, Zhou C, Chance B, Mohler ER 3rd, Yodh AG: Time-dependent blood flow and oxygenation in human skeletal muscles measured with noninvasive near-infrared diffuse optical spectroscopies. J Biomed Opt 2005;10:024027.

19 Wolf U, Wolf M, Choi JH, Paunescu LA, Safonova LP, Michalos A, Gratton E: Mapping of hemodynamics on the human calf with near infrared spectroscopy and the influence of the adipose tissue thickness. Adv Exp Med Biol 2003;510:225-230.

20 Shang Y, Zhao Y, Cheng R, Dong L, Irwin D, Yu G: Portable optical tissue flow oximeter based on diffuse correlation spectroscopy. Opt Lett 2009:34:3556-3558.

21 Health directorate of the Canton of Zurich: Rupture of the anterior cruciate ligament: surgical or conservative treatment? Report, June 30, 2009. 